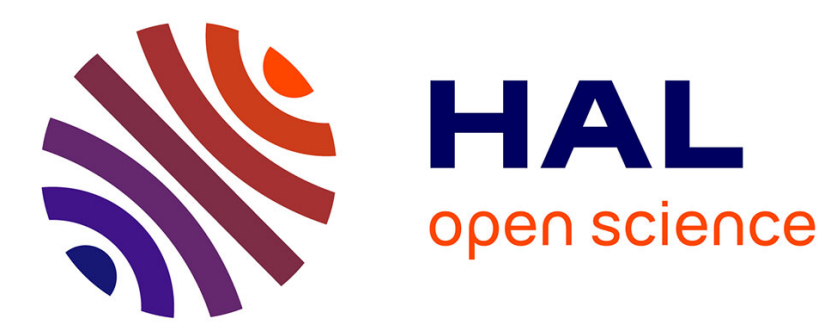

\title{
Qualitative Spatial Reasoning in RCC8 with OWL and SWRL
}

Stella Marc-Zwecker, François de Bertrand de Beuvron, Cecilia Zanni-Merk, Florence Le Ber

\section{- To cite this version:}

Stella Marc-Zwecker, François de Bertrand de Beuvron, Cecilia Zanni-Merk, Florence Le Ber. Qualitative Spatial Reasoning in RCC8 with OWL and SWRL. KEOD 2013 - International Conference on

Knowledge Engineering and Ontology Development, Sep 2013, Vilamoura, Portugal. hal-00914509

HAL Id: hal-00914509

https://hal.inria.fr/hal-00914509

Submitted on 5 Dec 2013

HAL is a multi-disciplinary open access archive for the deposit and dissemination of scientific research documents, whether they are published or not. The documents may come from teaching and research institutions in France or abroad, or from public or private research centers.
L'archive ouverte pluridisciplinaire HAL, est destinée au dépôt et à la diffusion de documents scientifiques de niveau recherche, publiés ou non, émanant des établissements d'enseignement et de recherche français ou étrangers, des laboratoires publics ou privés. 


\title{
Qualitative Spatial Reasoning in RCC8 with OWL and SWRL
}

\author{
Stella Marc-Zwecker ${ }^{1}$, François de Bertrand de Beuvron ${ }^{2}$, Cecilia Zanni-Merk ${ }^{2}$ and Florence Le Ber ${ }^{3}$ \\ ${ }^{1}$ ICube laboratory, BFO team, Strasbourg University, CNRS, France \\ ${ }^{2}$ ICube laboratory, BFO team, INSA de Strasbourg, CNRS, France \\ ${ }^{3}$ ICube laboratory, BFO team, Strasbourg University/ENGEES, CNRS, France \\ \{debeuvron, stella,merk\}@unistra.fr, florence.leber@engees.unistra.fr
}

Keywords: Qualitative Spatial Reasoning, RCC8, CM8, OWL, SWRL

Abstract: The Region Connection Calculus (RCC), and particularly its RCC8 subset, have been extensively studied
and used for qualitative spatial reasoning. Some sets of computational operations have also been defined for
topological relations, as the CM8 set, that allows to compute the RCC8 relationships on raster images. In this
paper, we propose a reified representation of the RCC8 spatial relationships and of the CM8 primitives, within
a lattice of concepts, implemented in OWL (Ontology Web Language) in order to help the interpretation of
urban satellite images. Our approach allows for a straightforward representation of concepts corresponding
to conjuctions or disjunctions of RCC8 spatial relationships, and thus offers the advantage to overcome some
drawbacks of the existing approaches in OWL, where spatial relations are represented as roles. Indeed, the
OWL language does not allow the expression of the disjunction or of the conjunction of roles. We can then
implement a reasoning on the RCC8 relationships, which in particular allows to compute the composition table
and its transitive closure. As the reification of roles precludes the use of role's properties, such as symmetry and
transitivity, we propose to implement RCC8 inferences through SWRL rules (Semantic Web Rule Language).

\section{Introduction}

The increasing availability of High Spatial Resolution satellite images is an opportunity to characterize and identify urban objects. Image analysis methods using object-based approaches relying on the use of domain knowledge are necessary to classify data. A major issue in these approaches is domain knowledge formalization and exploitation. The use of formal ontologies seems a judicious choice to deal with these issues. OWL (Grau et al., 2008) is a major language to implement such ontologies.

We have developed an ontology concerning urban objects (streets, houses, worker or residential housing, etc.) to assist the experts in their interpretation of satellite images and to be included, in the future, in a processing chain whose goal would be the automatic interpretation of the semantics of satellite images (Cravero et al., 2012).

In our ontology, the urban objects are defined by intension, that means they are derived from the conceptualization of a dictionary defined by expert geographers. However, to take into account the actual data from the image, it is necessary, also, to conceptualize spatial relations among the objects. The RCC 8 model
(Randell et al., 1992) defines eight basic topological relations between the regions from the image. Our proposition is to reify and implement these relations in OWL, and to use the SWRL rules for reasoning on spatial relation between objects and help the interpretation of complex structures on urban satellite images.

The reification of the topological relations is needed because of several drawbacks in OWL, as highlighted by several authors (Wessel, 2001). (Katz and Grau, 2005) presented one of the first attempts to represent RCC8 in OWL, proposing to extend the OWL reasoners with the functionality to operate with reflexive roles. (Hogenboom et al., 2010) claimed that OWL lacked essential features such as role negations, conjunctions, disjunctions and role inclusion axioms, to effectively represent RCC8. They proposed to use a more specific logic to express some of those constructs. (Jitkajornwanich et al., 2011) formalized 2D spatial concepts and operations into a spatial ontology, implemented as a plug-in for Protégé ${ }^{1}$. Unfortunately, the spatial relations retained by these authors are not the RCC 8 ones, although the querying possibilities using SWRL rules seem an interesting approach, close to ours. (Batsakis and Petrakis, 2011)

\footnotetext{
${ }^{1}$ http://protege.stanford.edu
} 
proposed an ontology for representing and reasoning over spatio-temporal information in OWL. The ontology enabled representation of static as well as of dynamic information, such as objects whose position evolves in time and space.It was built upon well established standards of the semantic web (OWL 2.0, SWRL), as the one we propose here. However, our approach focuses mainly on the definition of the RCC 8 relations and gives a complete specification of the TBox and ABox axioms and a minimal set of SWRL rules allowing, in this way, complete reasoning over any composition table.

The paper is organized as follows. After recalling the principles of the RCC 8 theory, we present a set of eight primitives, or computational operations (CM8), that are calculated by image processing routines, and that allow to establish the link with the actual data from the image (problem known as the semantic gap). In part 3, we present our proposition that is to reify the RCC8 relationships and the CM8 primitives in a lattice of concepts implemented in OWL. We then propose to use the SWRL rules (Horrocks et al., 2004) in order to reason about these concepts, and in particular, in order to deduce the composition table of the RCC8 relationships and its transitive closure. Part 4 is a conclusion.

\section{The RCC8 model for qualitative spatial reasoning}

\subsection{The RCC8 set of topological relations}

The set of eight topological relations defined in the RCC8 theory provides a conceptual basis for qualitative spatial reasoning (Randell et al., 1992). These relations are binary and they apply to a couple of regions $x$ and $y$ in a $n$-dimensional space. The elementary RCC8 relations are exhaustive and mutually exclusive, which means that any configuration of two spatial regions can be described by this set, and that if one of these relations is true, then the others are false. The eight RCC8 relations are:

- $E Q(x, y)$ " $x$ is identical to $y "$

- $\operatorname{TPP}(x, y)$ " $x$ is a tangential proper part of $y "$

- $T P P^{-1}(x, y) " y$ is a tangential proper part of $x$ "

- $\operatorname{NTPP}(x, y)$ " $x$ is a non-tangential proper part of $y$ "

- $N T P P^{-1}(x, y) " y$ is a non-tangential proper part of $x "$
- $P O(x, y)$ " $x$ partially overlaps $y "$

- $E C(x, y) " x$ is externally connected with $y$ "

- $D C(x, y)$ " $x$ is disconnected from $y$ "

The following mathematical operations are useful to make inferences on topological relations:

- the inverse relation of a relation $r$ is the relation $r^{-1}$ such that $\forall x, \forall y, r(x, y) \Leftrightarrow r^{-1}(y, x)$

- the relations $r 1$ and $r 2$ are disjoint if $\forall x, \forall y, r 1(x, y) \Rightarrow \neg r 2(x, y)$

- the complement of a relation $r$ is the relation $r c$ such that: $r$ and $r c$ are disjoint and $\forall x, \forall y, r(x, y) \vee$ $r c(x, y)$ is true.

- given three spatial regions $x, y, z$, and a pair of relations $r 1$ and $r 2$, the composition of $r 1(x, y)$ and $r 2(y, z)$ is the disjunction $r(x, z)$ of all the possible relations holding between $x$ and $z$.

The composition relation is particularly interesting because it allows the inferences of the possible relations between regions $x$ and $z$ from the known relations holding between the regions $x$ and $y$ on the one hand, and between the regions $y$ and $z$ on the other hand.

The rules of composition on the topological RCC8 relations are represented in composition tables (Randell et al., 1992).

\subsection{The CM8 set of computational operations}

In many situations, methods are needed to check topological relations on images or spatial databases. Computational operations have been defined in (Egenhofer, 1989; Clementini et al., 1993). They are based on the interiors and boundaries of spatial regions and are linked to formal models of topological relations.

In (Egenhofer, 1989), a method is defined to deal with vector data, that are mainly used in geographical information systems. A $n$ dimensional $x$ region is characterized by its $x^{\circ}$ interior set (same dimension) and its $\partial x$ boundary set $(n-1$ dimension). Intersecting these sets for two regions allows to define four operations: $\partial x \cap \partial y, x^{\circ} \cap y^{\circ}, \partial x \cap y^{\circ}$ et $x^{\circ} \cap \partial y$. A topological relation between two regions is then characterized in a unique way by the result values of the four operations. More recently, (Deng et al., 2007) proposed another set of operations based on the regions themselves, their interior and their boundary sets. The so-called ID-model uses set intersections, $x^{\circ} \cap y^{\circ}, \partial x \cap \partial y$, and set differences $x-y, y-x$.

These approaches are very interesting since they allow to express the RCC8 relations in terms of nec- 
essary and sufficient conditions on the regions. However a characterization of the regions in terms of interior and boundary is needed and this is a problem on raster images. When the raster representation is considered on its own, the boundary of a region can be defined by the pixels that are externally connected to the region but this definition does not meet the notion of connection that underlies RCC8 relationships: indeed, two disconnected regions may share a boundary point and thus could be considered as connected.

In (Le Ber and Napoli, 2003) the boundary is defined by abstract pixels, standing across four real pixels, as shown in Figure 1. The boundary intersection of two regions is then easily obtained, but relies on two images, the original image -containing the regions- and the boundaries image. Based on this representation four computational operations were introduced: the intersection of the interior sets, $x^{\circ} \cap y^{\circ}$; the intersection of the boundary sets, $\partial x \cap \partial y$; the two differences of the interior sets, $x^{\circ}-y^{\circ}$ and $y^{\circ}-x^{\circ}$.

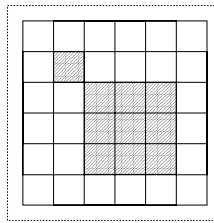

(a)

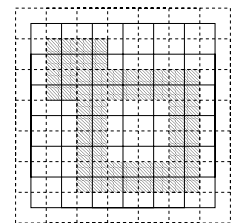

(b)

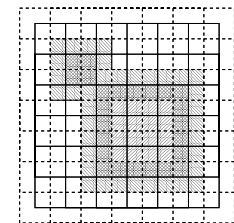

(c)
Figure 1: Defining the boundary of a region: (a) the interior of a region is made of real pixels (hatched); (b) the boundary of the region is made of abstract pixels standing across the real pixels (inverse hatched); (c) the combination of the interior and the boundary (Le Ber and Napoli, 2003).

From these four operations were derived the eight following conditions, called CM8 primitives (Le Ber and Napoli, 2003):

- $x^{\circ}-y^{\circ}=\emptyset, x$ is a part of $y$, denoted by $P(x, y)$

- $x^{\circ}-y^{\circ} \neq \emptyset, x$ is not a part of $y$, denoted by $N P(x, y)$

- $y^{\circ}-x^{\circ}=\emptyset, x$ contains $y$, denoted by $P^{-1}(x, y)$

- $y^{\circ}-x^{\circ} \neq \emptyset, x$ does not contain $y$, denoted by $N P^{-1}(x, y)$

- $x^{\circ} \cap y^{\circ}=\emptyset, x$ is discrete $y$, denoted by $D R(x, y)$

- $x^{\circ} \cap y^{\circ} \neq \emptyset, x$ overlaps $y$, denoted by $O(x, y)$

- $\partial x \cap \partial y=\emptyset, x$ does not share a boundary with $y$, denoted by $N A(x, y)$

- $\partial x \cap \partial y \neq \emptyset, x$ shares a boundary with $y$, denoted by $A(x, y)$

The CM8 primitives are expressed in terms of the RCC8 relationships, and vice versa, as shown in Table 1 . Indeed, this table can be interpreted in the following way :

- according to the lines: any RCC8 relation is expressed as a conjunction of CM8 primitives. For
Table 1: Correspondence between the RCC8 relationships and the CM8 primitives

\begin{tabular}{l|cccccccc} 
& $P$ & $N P$ & $P^{-1}$ & $N P^{-1}$ & $D R$ & $O$ & $N A$ & $A$ \\
\hline$E Q$ & 1 & 0 & 1 & 0 & 0 & 1 & 0 & 1 \\
$N T P P$ & 1 & 0 & 0 & 1 & 0 & 1 & 1 & 0 \\
$T P P$ & 1 & 0 & 0 & 1 & 0 & 1 & 0 & 1 \\
$N T P P^{-1}$ & 0 & 1 & 1 & 0 & 0 & 1 & 1 & 0 \\
$T P P^{-1}$ & 0 & 1 & 1 & 0 & 0 & 1 & 0 & 1 \\
$P O$ & 0 & 1 & 0 & 1 & 0 & 1 & 0 & 1 \\
$E C$ & 0 & 1 & 0 & 1 & 1 & 0 & 0 & 1 \\
$D C$ & 0 & 1 & 0 & 1 & 1 & 0 & 1 & 0
\end{tabular}

instance, $E Q \Leftrightarrow P \wedge P^{-1} \wedge O \wedge A$, means that two regions $x$ and $y$ are equal, if and only if, $x$ is a part of $y$, AND $y$ is a part of $x$, AND $x$ and $y$ overlap, AND the intersection between the boundaries of $x$ and $y$ is non empty.

- according to the columns : any CM8 primitive is expressed as a disjunction of RCC8 relations. For instance, $P \Leftrightarrow E Q \vee N T P P \vee T P P$, means that $x$ is a part of $y$, if and only if, $x$ is equal to $y$, OR $x$ is a tangential proper part of $y, \mathrm{OR} x$ is a nontangential proper part of $y$.

\section{Implementation of a qualitative spatial reasoning using $O W L$ and SWRL}

As shown in section 1, the existing approaches for reasoning on spatial qualitative relations outline the lack of expressiveness of OWL for the negation, conjunction or disjunction of roles. However, these features are essential to implement the composition rules of the RCC8 relations. In this section, we first present the principle of reification and we show how it is used to implement a lattice of RCC8 and CM8 concepts. We then introduce a set of SWRL rules, allowing to compute the relation composition.

\subsection{Reification of spatial relations}

Reification is widely used in conceptual modelling. Reifying a relation means viewing it as an entity, that describes the relation's characteristics.

Although RCC8 relationships are simple binary relations, there are other spatial relations that require additional attributes. A simple example, used in our satellite image analysis application, is the distance between objects (either minimal distance or distance between barycentres) that requires a numeric value. A 
coherent processing of all the spatial relations will require to reify them all.

Moreover, reasoning on RCC8 relationships causes the creation of new relations, which are built from the eight basic RCC8 relations, by applying conjunction or disjunction. However, conjunction or disjunction of roles are beyond the expressive power of OWL. This is why many specific extensions have been proposed, as shown in section 1 .

In our approach, spatial relations are represented by concepts of the ontology. Let us note $S R$, the top concept of the hierarchy of spatial relations. It is straightforward to combine the concepts of this hierarchy by using the complete set of logic operators (and, or, not), which are already available in the $\mathcal{A} \mathcal{L C}$ description logics. Each instance of the $S R$ concept represents a relation between two geographical objects. As RCC8 relations are generally not symmetric, we associate two distinct functional roles to the $S R$ concept: a spatial relation takes place from a first geographical object to a second geographical object.

\section{$S R \sqsubseteq \neg G e o O b j e c t$}

(objects and spatial relations are disjoint)

$$
S R \sqsubseteq(=1 \text { from.GeoObject }) \sqcap(=1 \text { to.GeoObject })
$$

(any spatial relation associates exactly two objects)

We now define the general $R C C 8$ concept, included in $S R$, which subsumes all the concepts resulting from the combination of the RCC8 basic relations.

The eight elementary RCC8 relations form a complete and disjoint partition of the RCC8 concept.

The CM8 primitives are defined as disjunctions of the RCC 8 basic relations. For example, the primitives denoted by $P$ (inclusion) and $O$ (overlapping) are expressed in the following way:

$$
\begin{aligned}
& P \equiv E Q \sqcup N T P P \sqcup T P P \\
& O \equiv E Q \sqcup N T P P \sqcup T P P \sqcup N T P P^{-1} \sqcup T P P^{-1} \sqcup P O
\end{aligned}
$$

The reification of spatial relations among objects provokes no particular problems, although the notation is slightly more complex. For example, we want to state that a workers housing estate consists of a set of adjoining houses within an urban area.

$$
\text { uArea } \sqsubseteq \text { GeoObject, House } \sqsubseteq \text { GeoObject }
$$

Without reification ( $\mathrm{Pr}$ and $\mathrm{ECr}$ are roles corresponding to the $P$ and $E C$ spatial relations):

$$
\begin{aligned}
\text { wHousing } \equiv & \text { GeoObject } \sqcap \exists \text { Pr.uArea } \square \\
& \forall \text { Pr }^{-1} .(\neg \text { House } \sqcup \exists \text { ECr.House })
\end{aligned}
$$

After reification ( $P$ and $E C$ are $R C C 8$ subconcepts corresponding to the $P$ and $E C$ spatial relations):

$$
\begin{aligned}
\text { wHousing } \equiv & \text { GeoObject } \sqcap \\
& \exists \text { from }^{-1} .(P \sqcap \exists \text { to.uArea }) \sqcap \\
& \forall t o^{-1} \cdot(\neg P \sqcup \forall \text { from. }(\neg \text { House } \sqcup \\
& \exists t o^{-1} .(\text { EC } \boxminus \exists \text { from.House })
\end{aligned}
$$

The reified expressions are cumbersome, but the translation from the non-reified form to the reified form can be easily automated.

At ABox level, additional individuals must be created to represent the reified spatial relation between every couple of objects (see Algorithm 2).

Figure 2 gives the ABox corresponding to an urban area containing one workers housing $w h 1$, containing two adjacent houses $h 1$ and $h 2 . s r_{i}$ individuals represent the reified spatial relations. Undefined spatial relations (of RCC8 type) are omitted for brevity.

Our representation allows a precise description of spatial relationships between individuals. For example:

- $o_{1}:\left(=2\right.$ from $\left.^{-1} \cdot \neg D C\right)$, exactly two objects are connected with $o_{1}$. The precise nature of the connection $(E Q, N T T P, P \ldots)$ is unknown (for exemple, the image analysis system has not been able to determine it)

- $o_{2}:\left(<=3\right.$ from $\left.^{-1} . O\right) \sqcap \exists$ from $^{-1} . P$, at most three objects overlap with $o_{2}$, at least one of them contains $o_{2}$.

We have presented so far the good properties of our model, deliberately leaving aside its disadvantages, some of which are significant. First, there is no way to express the correspondence between a spatial relation and its inverse: in the previous example, we stated that house $h 1$ was adjacent $(E C)$ to house $h 2$, But we cannot deduce the fact, however obvious, that $h 2$ is adjacent to $h 1$. If spatial relations were represented by roles, this problem could be solved by declaring the role as symmetric in OWL.

There is also no way to handle transitivity: in our example, the house $h 1$ is included $(P)$ in the workers' housing $w h 1 ; w h 1$ itself is included in the urban area $u a 1$. No valid inference will deduce the obvious fact that $h 1$ is included in $u a 1$ (transitivity of inclusion). These disadvantages can be overridden by the use of SWRL rules.

\subsection{Using SWRL Rules to simulate role properties}

Two types of rules must be defined to reflect respectively inverse relations and the composition table. The 


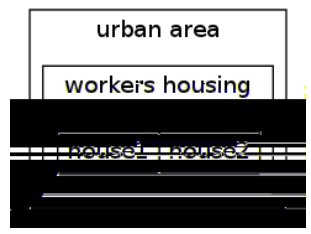

h1: House, h2: House, wh1 : wHousing, ua $1:$ uArea $s r_{1}: E C, f r o m\left(s r_{1}, h 1\right), t o\left(s r_{1}, h 2\right)$

$s r_{2}: P, \operatorname{from}\left(s r_{2}, h 1\right), t o\left(s r_{2}, w h 1\right)$

$s r_{3}: P, \operatorname{from}\left(s r_{3}, h 2\right), t o\left(s r_{3}, w h 1\right)$

sr4 : P, from $(s r 4, w h 1), \operatorname{to}(s r 4, u a 1)$

Figure 2: An ABox for an urban area containing only two houses within a worker's housing

inverse relations were not a problem before reification, since OWL allows the definition of inverse, symmetric and transitive roles. Unfortunately, after reification, it is no longer possible to express the correspondences between a relation and its inverse in OWL. We therefore propose to use rules. As we have seen in section 2, it is straightforward to express RCC8 inverse relations in First Order Logic:

$$
\begin{gathered}
\forall o_{1} \forall o_{2} E Q\left(o_{1}, o_{2}\right) \Rightarrow E Q\left(o_{2}, o_{1}\right) \\
\text { (symmetric relation) } \\
\forall o_{1} \forall o_{2} T P P\left(o_{1}, o_{2}\right) \Rightarrow T P P^{-1}\left(o_{2}, o_{1}\right):
\end{gathered}
$$$$
\text { (explicit inverse relation) }
$$

After reification, spatial relations become objects of the logical universe, and the above rules must be expanded as:

$$
\begin{array}{r}
\forall r_{1} \forall r_{2} \forall o_{1} \forall o_{2} E Q\left(r_{1}\right) \wedge \operatorname{from}\left(r_{1}, o_{1}\right) \wedge \operatorname{to}\left(r_{1}, o_{2}\right) \wedge \\
\operatorname{from}\left(r_{2}, o_{2}\right) \wedge \operatorname{to}\left(r_{2}, o_{1}\right) \Rightarrow E Q\left(r_{2}\right) \\
\forall r_{1} \forall r_{2} \forall o_{1} \forall o_{2} T P P\left(r_{1}\right) \wedge \operatorname{from}\left(r_{1}, o_{1}\right) \wedge \operatorname{to}\left(r_{1}, o_{2}\right) \wedge \\
\operatorname{from}\left(r_{2}, o_{2}\right) \wedge \operatorname{to}\left(r_{2}, o_{1}\right) \Rightarrow T P P^{-1}\left(r_{2}\right)
\end{array}
$$

The same principle is used to represent the RCC 8 composition of relations. For example, the result of composing $P O$ with $T P P$ is either ${ }^{2} T P P, N T P P$ or $P O$. After reification, this should be represented by:

$$
\begin{aligned}
& \forall r_{1} \forall r_{2} \forall r_{3} \forall o_{1} \forall o_{2} \forall o_{3} \\
& P O\left(r_{1}\right) \wedge \operatorname{from}\left(r_{1}, o_{1}\right) \wedge \operatorname{to}\left(r_{1}, o_{2}\right) \wedge \\
& T P P\left(r_{2}\right) \wedge \operatorname{from}\left(r_{2}, o_{2}\right) \wedge \operatorname{to}\left(r_{2}, o_{3}\right) \wedge \\
& \operatorname{from}\left(r_{3}, o_{1}\right) \wedge \operatorname{to}\left(r_{3}, o_{3}\right) \\
& \Rightarrow T P P\left(r_{3}\right) \vee N T P P\left(r_{3}\right) \vee P O\left(r_{3}\right)
\end{aligned}
$$

SWRL is based on Horn clauses. However, the head of a Horn clause can not be a disjunction. So,

\footnotetext{
${ }^{2} P O(x, y) \wedge T P P(y, z) \Rightarrow T P P(x, z) \vee N T P P(x, z) \vee$
}

in order to represent disjunctions, a new concept is created each time a specific disjunction appears in the conclusion of a composition rule. For the rule example above :

$$
T P P \_N T P P \_P O \equiv T P P \sqcup N T P P \sqcup P O
$$

The SWRL rule simply becomes (in human readable SWRL syntax):

$$
\begin{aligned}
& P O\left(? r_{1}\right) \wedge \operatorname{from}\left(? r_{1}, ? o_{1}\right) \wedge t o\left(? r_{1}, ? o_{2}\right) \wedge \\
& \operatorname{TPP}\left(? r_{2}\right) \wedge \operatorname{from}\left(? r_{2}, ? o_{2}\right) \wedge t o\left(? r_{2}, ? o_{3}\right) \wedge \\
& \operatorname{from}\left(? r_{3}, ? o_{1}\right) \wedge t o\left(? r_{3}, ? o_{3}\right) \\
& \Rightarrow T P P \_N T P P \_P O\left(? r_{3}\right)
\end{aligned}
$$

More formally, we need to determine the set of rules to ensure complete reasoning over the composition table. We will denote by $\mathcal{E} \mathcal{R}$ the set of elementary spatial relations and by $\mathcal{R}=2^{\mathcal{E} R}$ the power set of $\mathcal{E} R$, where each set in $\mathcal{R}$ denotes a disjunction of elementary spatial relations. We will note $e r_{i} \in \mathcal{E} \mathcal{R}$ the elementary spatial relations, and $s r_{i} \in \mathcal{R}$ the disjunctive spatial relations. An elementary composition table $\mathcal{E} C \mathcal{T}$ is a function $\mathcal{E} C \mathcal{T}: \mathcal{E} R \times \mathcal{E} R \rightarrow \mathcal{R}$. A generalized composition table $C \mathcal{T}: R \times R \rightarrow R$ can be derived from $\mathcal{E} \mathcal{C} \mathcal{T}$ by :

$$
\mathcal{C} \mathcal{T}\left(s r_{1}, s r_{2}\right)=\bigsqcup_{e r_{1} \in s r_{1}} \bigsqcup_{e r_{2} \in s r_{2}} \mathcal{E} \mathcal{C}\left(e r_{1}, e r_{2}\right)
$$

A subset $S \subseteq \mathcal{R}$ is closed under a composition table $\mathcal{C} \mathcal{T}$ if $\forall s r 1, s r 2 \in \mathcal{S}: \mathcal{C} \mathcal{T}(s r 1, s r 2) \in \mathcal{S}$. From any subset $S \subseteq \mathcal{R}$, it is straightforward to compute its closure $\mathcal{C}_{C \mathcal{T}}(S)$ by repeatedly applying $C \mathcal{T}$ rules.

The generalized composition table $C \mathcal{T}$ has to be translated into a set of SWRL rules, where each rule is a triple $\left[s r_{1}, s r_{2}, s r_{3}=\mathcal{C} \mathcal{T}\left(s r_{1}, s r_{2}\right)\right]$. Since each $s r_{i}$ is a disjunction of elementary RCC8 relations, some rules in $\mathcal{C} \mathcal{T}$ may be logicaly redundant. More precisely, a rule $r 1=\left[s r_{1}^{1}, s r_{2}^{1}, s r_{3}^{1}\right]$ is more general than a rule $r 2=\left[s r_{1}^{2}, s r_{2}^{2}, s r_{3}^{2}\right]$, noted $r 2 \preceq r 1$, if and only if :

$$
r 2 \preceq r 1 \Leftrightarrow\left(s r_{1}^{2} \subseteq s r_{1}^{1} \wedge s r_{2}^{2} \subseteq s r_{2}^{1} \wedge s r_{3}^{1} \subseteq s r_{3}^{2}\right)
$$

We will denote by $\mathcal{G}_{\preceq}(\mathcal{C} \mathcal{T})$ the set of greatest elements of $\mathcal{C} \mathcal{T}$ for the partial order $\preceq$.

Depending on the application, one may want to consider only a subset $\mathcal{U} \mathcal{R} \subseteq \mathcal{R}$ of spatial relations. In our approach, $\mathcal{U} R$ corresponds to the set of RCC8 relationships augmented by the set of CM8 primitives.

Therefore, given a qualitative reasoning system defined by a set of elementary relations $\mathcal{E} \mathcal{R}$ and an elementary composition table $\mathcal{E} \mathcal{C} \mathcal{T}$, the set of rules for a user defined subset $\mathcal{U R} \subseteq 2^{\mathcal{E} R}$ is given by:

$$
\mathcal{G}_{\preceq}\left(\mathcal{C} \mathcal{T}\left\lceil_{\mathcal{C}_{\mathcal{C T}}(\mathcal{U R})}\right)\right.
$$


where $f \uparrow_{A}$ denotes the restriction of $f$ to the domain $A$. The corresponding algorithm is given in Algorithm 1.

In our application, a set of 94 SWRL rules is generated for the creation of the composition table and its transitive closure. It is interesting to note that the consideration of the CM8 primitives slightly decreases the number of SWRL rules generated (100 rules for a lattice with only the RCC8 relationships). Indeed, the introduction of the CM8 primitives allows the factorisation of some rules (according to the $\preceq$ partial order between the rules).

For example, in the initial RCC8 composition table, there are three rules stating that, for three spatial objects $o_{1}, o_{2}, o_{3}$, if $o_{1}$ is disconnected (DC) from $o_{2}$ and $o_{3}$ is either identical $(E Q)$, a tangential $\left(T P P i^{3}\right)$ or non-tangential $(N T P P i)$ proper part of $o_{2}$, then $o_{1}$ is disconnected from $o_{3}$. As $P i \Leftrightarrow$ $E Q \vee N T P P i \vee T P P i$, these three rules will be factored into the single SWRL rule of Figure 3.

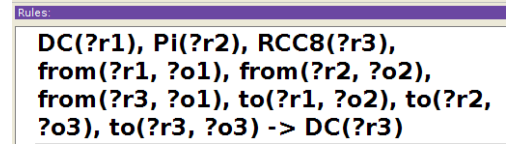

Figure 3: a SWRL rule for the composition of DC and Pi

Figure 4 presents the lattice of concepts containing the eight $\mathrm{RCC} 8$ relations, the $\mathrm{CM} 8$ primitives allowing to compute RCC8 relations from the image, as well as all intermediate concepts generated by the rules that implement the transitive closure of the composition table. We can note that the lattice's hierarchy reflects the correspondence between the RCC 8 relationships and the CM8 primitives (see Table 1) : a RCC8 relation is a conjunction of its CM8 ancestors (e.g. $E Q \Leftrightarrow P \wedge P I \wedge O \wedge A$ ), and a CM8 primitive is a disjunction of its RCC8 descendants (e.g. $P \Leftrightarrow E Q \vee N T P P \vee T P P)$.

\subsection{Reasoning}

Let us continue the example introduced in section 4.1 to show how our model can be effectively used to infer new information linking spatial reasoning to the domain ontology. The definition of a workers housing estate (wHousing) as a set of adjoining houses within an urban area is presented in Figure 5 using the Manchester OWL Syntax of Protégé. Suppose now that the wh 1 instance has not been associated with

\footnotetext{
${ }^{3}$ In general, for every spatial relation $S R, S R i$ represents its inverse.
}

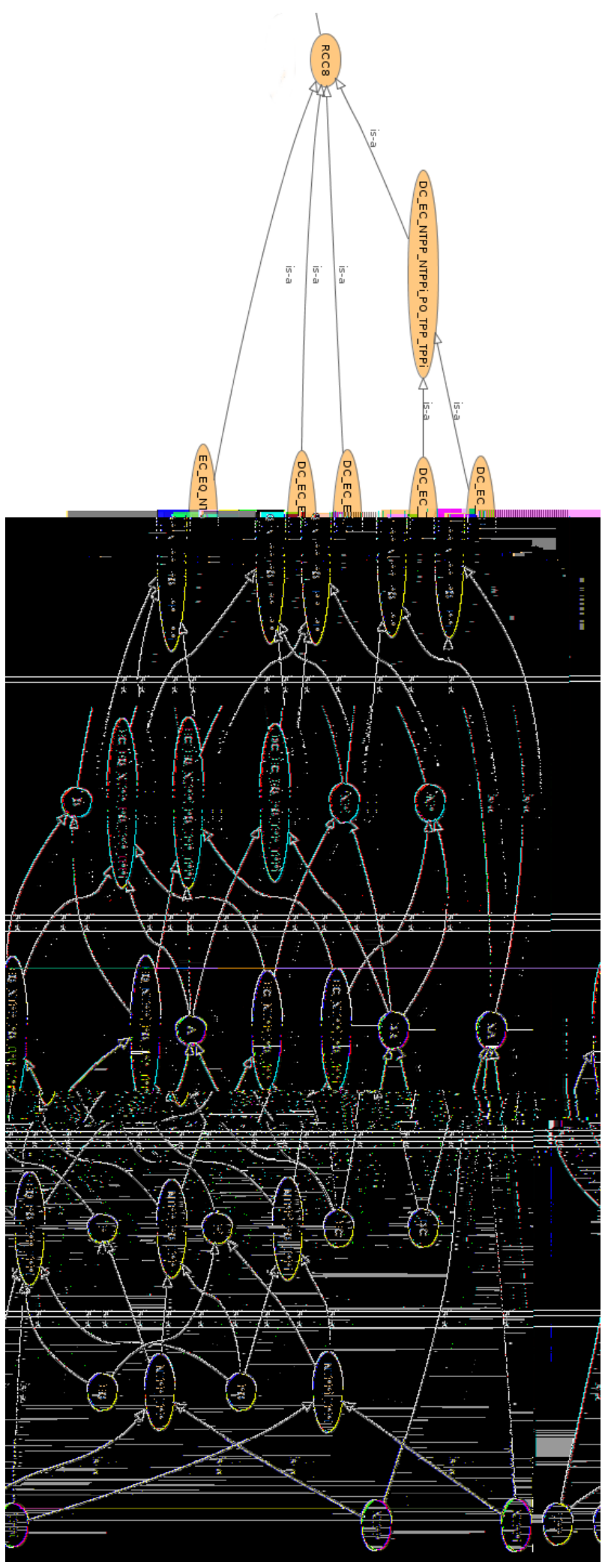

Figure 4: The lattice of the RCC8 spatial relations, with the CM8 primitives 


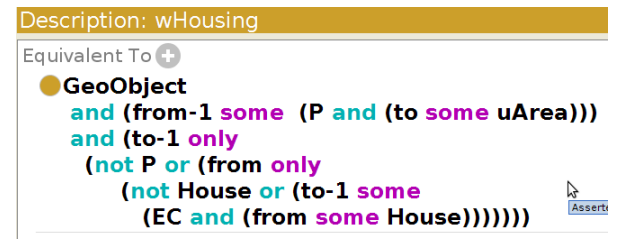

Figure 5: workers housing estate definition

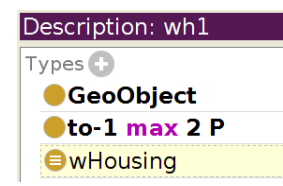

Figure 6: wh1 is inferred to be a workers housing estate

wHousing in the ontology ie :

$$
\begin{aligned}
& \text { h1 : House, h2 : House, ua } 1: \text { uArea } \\
& \text { wh1 : GeoObject, }
\end{aligned}
$$

Any sound OWL reasoner will not recognize wh1 as an instance of wHousing. This negative result would be surprising if working with an object oriented database, but it is consistent with the OWL-DL semantics which is based on the open world assumption. Actually, the OWL-DL reasoner infers that $h 1$ and $h 2$ are adjoining houses, but with the open world assumption, the existence of another house $h 3$ cannot be excluded. Indeed, if we had postulated that $h 3$ was within $w h 1$ but not adjacent to $h 1$ nor $h 2$, then wh1 would not have been a wHousing.

Therefore in our example, the image recognition software must state that the houses $h 1$ and $h 2$ are the only objects included in wh1. For this purpose, the wh1 instance description must be extended with this knowledge by a cardinality constraint (see Figure 6). The reasoner (Hermit 1.3.6) now correctly infers that wh 1 actually is a workers housing estate.

Suppose we introduce a third house in our workers housing:

$$
\begin{aligned}
& \text { isolatedHouse : House } \\
& \text { sr5 : P, from }(\text { sr5, isolatedHouse }), \text { to }(\operatorname{sr} 5, \text { wh } 1)
\end{aligned}
$$

Remember that reasoning in OWL is under the open world assumption: our isolated house is not explicitly adjacent to another house in the ABox, but neither is it explicitly defined that it is not adjacent to $h 1$ or $h 2$, or even to any other house which is not defined in the ABox. Therefore, the ABox is still consistent, even if we have required that all the houses must have at least one adjacent house in the definition of the wHousing concept. Fortunately, cardinality constraints on roles allow to simulate closed world reasoning. If we are sure that the house is isolated, we can for example add in the ABox:

$$
\text { isolatedHouse }: \leq 0 \text { from }^{-1} \text {.EC }
$$

This time the ABox is inconsistent.

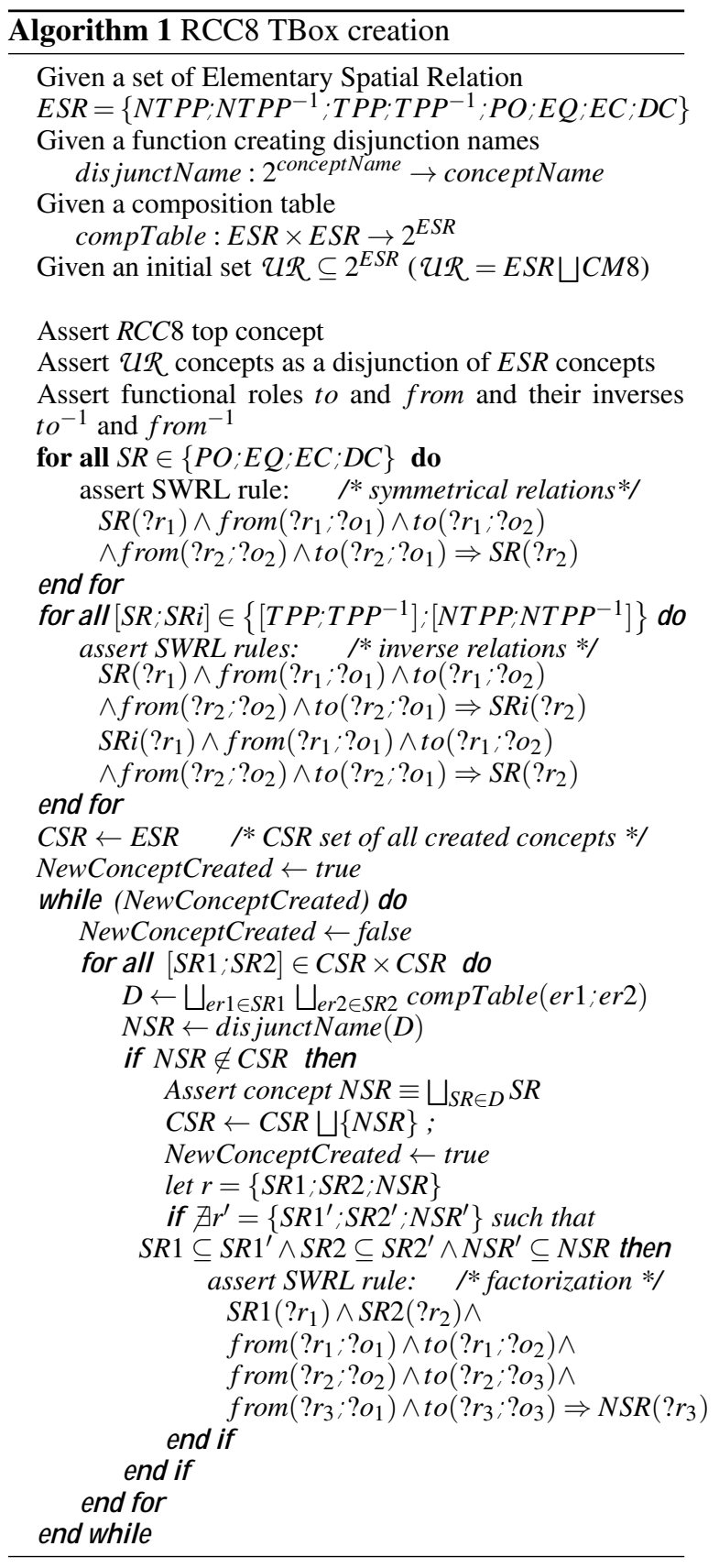

\section{Conclusions}

In this paper we have described the implementation of a qualitative spatial reasoning on topological RCC8 relationships, based on OWL. 


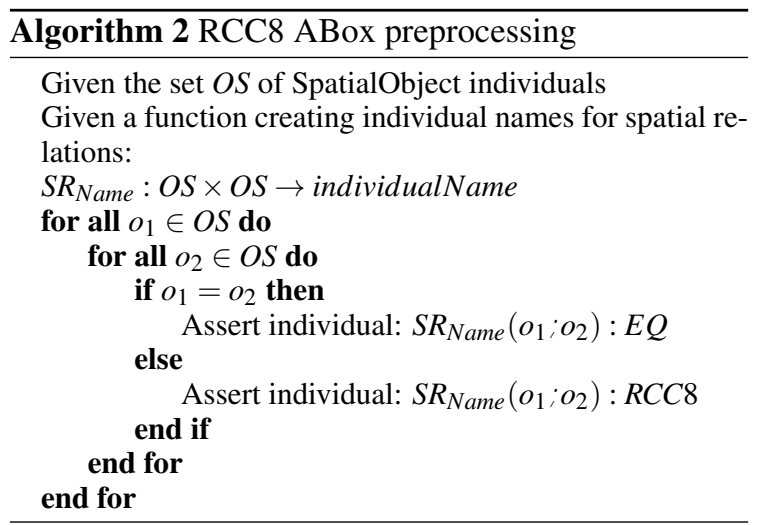

Our approach copes with the lack of expressiveness of OWL for implementing the negation, conjunction or disjunction of roles. This approach relies on the reification of roles, and is completed by the use of SWRL rules to overcome the loss, due to reification, of some roles properties in OWL, such as symmetry or transitivity.

A disadvantage of this approach is the additional complexity induced by the process of reification, which causes the creation of $n^{2}$ instances of spatial relations for $n$ geographic objects. Thus, a reasoner that would be optimized for executing SWRL rules involving many instances would be required.

However, the interest of our proposal is twofold. On the one hand, we have demonstrated the feasibility of the implementation based on OWL and SWRL, of a complete reasoning for calculating the composition table of the RCC8 relationships and its transitive closure. On the other hand, our model integrates the expression of the RCC8 relationships in terms of the CM8 computational primitives: this opens interesting perspectives for the extraction of the topological relations existing among image objects, in the context of satellite images recognition. Other sets of primitives could be included as well.

Furthermore, we plan to implement the CM8 computational primitives, and to integrate them in the image classification software that has been developed in our research team (the MUSTIC platform ${ }^{4}$ ).

\section{REFERENCES}

Batsakis, S. and Petrakis, E. G. M. (2011). SOWL: A Framework for Handling Spatio-temporal Information in OWL 2.0. In RuleML Europe, pages 242-249.

Clementini, E., Felice, P. D., and Oosterom, P. v. (1993).

\footnotetext{
${ }^{4}$ http://icube-bfo.unistra.fr/fr/index.php/ Plateformes
}

A small set of formal topological relationships suitable for end-user interaction. In Proceedings of the Third International Symposium on Advances in Spatial Databases, SSD 93, pages 277-295, London, UK. Springer-Verlag.

Cravero, M., Zanni-Merk, C., de Bertrand de Beuvron, F., and Marc-Zwecker, S. (2012). A Description Logics Geographical Ontology for Effective Semantic Analysis of Satellite Images. In 16th International Conference on Knowledge-Based and Intelligent Information \& Engineering Systems, KES2012, volume 243, pages 1573-1582. IOS Press.

Deng, M., Cheng, T., Chen, X., and Li, Z. (2007). Multilevel Topological Relations Between Spatial Regions Based Upon Topological Invariants. GeoInformatica, 11:239-267.

Egenhofer, M. J. (1989). A Formal Definition of Binary Topological Relationships. In Litwin, W. and Schek, H., editors, Foundations of Data Organization and Algorithms, FODO 1989, Paris, France, LNCS 367, pages 457-472. Springer Verlag.

Grau, B. C., Horrocks, I., Motik, B., Parsia, B., PatelSchneider, P., and Sattler, U. (2008). OWL 2: The next step for OWL. Web Semantics: Science, Services and Agents on the World Wide Web, 6(4):309 - 322. Semantic Web Challenge 2006/2007.

Hogenboom, F., Borgman, B., Frasincar, F., and Kaymak, U. (2010). Spatial Knowledge Representation on the Semantic Web. In Proceedings of the 2010 IEEE Fourth International Conference on Semantic Computing, ICSC'10, pages 252-259, Washington, DC, USA. IEEE Computer Society.

Horrocks, I., Patel-Schneider, P. F., Boley, H., Tabet, S., Grosof, B., and Dean, M. (2004). SWRL: A Semantic Web Rule Language Combining OWL and RuleML. W3c member submission, World Wide Web Consortium.

Jitkajornwanich, K., Elmasri, R., Li, C., and McEnery, J. (2011). Formalization of 2-D spatial ontology and OWL/Protégé realization. In Proceedings of the International Workshop on Semantic Web Information Management, SWIM'11, pages 9:1-9:7, New York, NY, USA. ACM.

Katz, Y. and Grau, B. C. (2005). B.C.: Representing Qualitative Spatial Information in OWL-DL. In Proceedings of OWL: Experiences and Directions.

Le Ber, F. and Napoli, A. (2003). Design and comparison of lattices of topological relations for spatial representation and reasoning. Journal of Experimental \& Theoretical Artificial Intelligence, 15(3):331-371.

Randell, D. A., Cui, Z., and Cohn, A. G. (1992). A spatial logic based on regions and connection. In Proceedings 3rd International Conference on Knowedge Representation and Reasoning.

Wessel, M. (2001). Obstacles on the Way to Qualitative Spatial Reasoning with Description Logics: Some Undecidability Results. In Proceedings of the International Workshop in Description Logics 2001 (DL2001), pages 96-105. 